\title{
GI-MS45-P02 | STRUCTUREFINDER
}

Kratzert, Daniel (Albert-Ludwigs-Universität Freiburg, Freiburg, GER)

Presented is a new computer program called StructureFinder. It creates a database of crystallographic structures on a computer and makes them searchable. The program can search for various properties: Unit cell, free text, creation date, included atom types and space group. To build the database, StructureFinder collects all computer information files (cif file format) below certain directories on a hard disk. The containing information is stored into a SQLite database. The database can be accessed by two different interfaces. Either, a stand-alone Qt program to install on a single computer or a web interface to be accessed by a whole work group.

StructureFinder greatly enhances the ability to find old structures in huge collections of crystallographic datasets. The program can easily handle more than 10.000 structures. It can be downloaded at [1].

[1] https://www.xs3.uni-freiburg.de/research/structurefinder 